\title{
Intraneural Injection in Regional Anesthesia: What Does the Literature Tell Us?
}

\author{
Robert S. Weller
}

Published online: 14 August 2013

(C) Springer Science+Business Media New York 2013

\begin{abstract}
The contemporary use of ultrasound-guidance for peripheral nerve block allows for direct imaging of the relationship of needle-to-nerve. In the past it was presumed a block needle did not penetrate the nerve, but it is now apparent that unintentional, and even intentional, needle placement deep to the outer epineurium may occur without pain, paresthesia, or stimulating motor response below $0.5 \mathrm{~mA}$. It remains controversial whether needle placement just outside the outer epineurium is effective in producing block, and less likely to contribute to postoperative neurologic symptoms than intentional intraneural needle placement which may produce faster onset of blockade, and sometimes more complete or longer blockade. Since extraneural, circumferential injection of local anesthetic is effective, and since current ultrasound and other technology cannot reliably prevent an intraneural needle from penetrating a fascicle with a potential for nerve injury, the extraneural needle location is concluded to be a more appropriate location.
\end{abstract}

Keywords Intraneural $\cdot$ Nerve block $\cdot$ Postoperative neurologic symptoms · Ultrasound

\section{Introduction}

For decades, nerve localization methods for peripheral nerve block maintained the assumption that the needle was carefully being advanced close to, but not directly inside the target nerve. With paresthesia-elicitation, and later,

R. S. Weller $(\bowtie)$

Wake Forest School of Medicine, Medical Center Blvd,

Winston-Salem, NC 27157-1009, USA

e-mail: rweller@wakehealth.edu nerve stimulation techniques, the clinician expected there would be an indication that the needle was "too close," either by the report of pain with the former or motor response with a current below $0.2 \mathrm{~mA}$ with the latter. Breaching the epineurium was never intended, and there was confidence that careful needle advancement with continuous observation of the patient's response would guide the anesthesiologist to a "proper" needle placement just outside the epineurium [1]. The introduction of ultrasound in the late 1990s as a needle guidance technique for peripheral nerve block allowed for the direct observation of needle-to-nerve proximity for the first time. This led to the demonstration in animals that a needle could be placed directly through the epineurium, but the evoked motor response from this intraneural needle sometimes required stimulation current even greater than $1.0 \mathrm{~mA}$ [2]. In studies in patients, it was observed that needle-to-nerve contact did not necessarily result in motor response (especially at lower currents $<0.5 \mathrm{~mA}$ ) [3] or a sensation of paresthesia [4]. In an investigation of motor stimulating threshold in ultrasound-guided supraclavicular block, Bigeleisen et al. positioned a needle first outside, then inside the apparent epineurium surrounding the most superficial part of the brachial plexus. They showed that although $87 \%$ of patients had lower stimulating threshold intraneural compared to extraneural, $10 \%$ of patients with intraneural needle placement required a stimulating current $>0.5 \mathrm{~mA}$ for a motor response [5]. Further, there is evidence that for some nerve blocks, particularly popliteal, that the standard nerve stimulation technique using an endpoint of $0.5 \mathrm{~mA}$ may result in needle puncture of the outer epineurium over $90 \%$ of the time [6*].

All of these observations culminated in the current controversy: whether it is in fact advantageous for the needle to intentionally be advanced through the 
epineurium, with respect to onset and quality of nerve block, or whether this "aggressive" needle placement is unnecessary and increases the potential to injure the nerve and result in postoperative neurologic symptoms (PONS).

\section{Anatomy of the Peripheral Nerve}

Before discussing "intraneural," it is crucial to review the histology of the peripheral nerve and to define exactly what is meant by the term "intraneural." From landmark neuroanatomic studies by Sunderland beginning in the 1950s, the topography of the sciatic, median, and other nerves was well characterized. The axons of a peripheral nerve, with cell bodies in the segmental dorsal root ganglia, are tightly organized in discrete bundles or fascicles, whose outer dense connective tissue layer is called the perineurium. The inner connective tissue separating and supporting the individual axons (and including those axons) is called the endoneurium, and the diffusion barrier presented by the perineurium creates a biochemically stable environment within the perineurium to maintain homeostasis of the axons. The perineurium is sometimes referred to as the bloodnerve barrier [7] .

It was demonstrated, also nearly 50 years ago, that disruption of the perineural barrier by a needle leads to axonal degeneration. The dense barrier of the perineurium results in the endoneurium displaying low compliance, and a penetrating needle injury to the axons is exacerbated by injection of solution. Injection of local anesthetic (LA) into the fascicle, particularly with epinephrine, produced even greater degrees of injury than injection of saline alone [8]. Needle bevel type was also shown to influence the degree of fascicular injury, with sharp needles more likely to penetrate the perineurium than shallow bevel needles, although once a shallow bevel needle penetrated the fascicle, it produced more axonal damage than the sharp bevel [9]. More recent animal studies in dogs showed that intraneural LA injection requiring high pressures ( $>20 \mathrm{psi})$ and presumed to be intrafascicular because of the low compliance, resulted in persistent neurologic dysfunction [10]. It remains indisputable that needle penetration of the perineurium and into a fascicle of a peripheral nerve should be avoided, since it carries a high likelihood of injury to that fascicle.

Between the fascicles, however, a variable amount of loose connective tissue is present which contains blood vessels, fat, and some communicating nerve fibers passing from one fascicle to another, or exiting (efferent) or entering (afferent) a nerve fascicle. The condensation of this connective tissue at the surface of the nerve is called the outer epineurium, with the connective tissue between fascicles called the inner epineurium. The outer epineurium does not have the tight barrier properties of the perineurium, and the epineurium as well as the spiraling of fascicles allows for movement and stretch of peripheral nerves before a longitudinal compliance limit is reached.

Finally, there may be further layers of connective tissue outside of the epineurium, such as the perimysium of surrounding muscles such as the middle and anterior scalene, or the common connective tissue layer surrounding the common peroneal and the tibial nerves, each with its own outer epineurium, which some have called the "paraneurium" [11].

\section{Defining Intra- and Extra-neural}

Needle advancement, then, through the outer epineurium, or even the perineurium, could change "all" to "both" be referred to as "intraneural," so one must be quite clear in the meaning of the term. For the purpose of this monograph, intraneural will be defined as either "intraneuralintrafascicular," which is always to be avoided, or "intraneural-extrafascicular," which remains controversial as an intended needle location. A needle outside the outer epineurium will be called "extraneural."

To reiterate the debate, it is clear that intraneuralintrafascicular injection should be avoided, but the question remains: is it optimal to position the needle and inject the LA either extraneural, or instead position it intraneuralextrafascicular for successful neural blockade with an acceptably low risk of PONS? It must be emphasized that PONS is not always due to nerve block and needle trauma or local anesthetic injection/toxicity; it can occur following many procedures on the extremities without any block being performed [12], and appears to be more likely in patients with preexisting neuropathy [13]. With respect to potential injury caused by our nerve block techniques, though, anesthesiologists would like to be confident that their procedures carry the lowest chance of causing direct needle injury or PONS while still having a high success rate to provide the benefits of regional anesthesia to their patients.

\section{What is the Evidence that the Extraneural Location for LA Injection is Successful?}

Ultimately, for successful nerve block, the diffusion of LA according to its concentration gradient and into target axons must produce a sufficient intraneural minimum blocking concentration $\left(\mathrm{C}_{\mathrm{m}}\right)$ to block sodium channels and action potential propagation. Some degree of longitudinal spread of LA along a myelinated nerve is required for reliable block since a critical number of adjacent nodes of 
Ranvier along the axon must be blocked to extinguish action potentials [14]. The volume of local anesthetic for ultrasound-guided, extraneural nerve block has been shown to be much lower than volumes historically used with nerve stimulator or paresthesia-guided techniques. In axillary block, a dose-finding study demonstrated that extraneural injection of volumes as low as $1 \mathrm{~mL}$ is successful in producing complete blockade of nerves in the axilla [15]. In a dose-finding study for mid-femoral, ultrasound-guided sciatic block, investigators found an $\mathrm{ED}_{99}$ of $0.10 \mathrm{~mL} / \mathrm{mm}^{2}$ cross sectional area of the sciatic nerve (median area $56 \mathrm{~mm}^{2}$ ), but complete sensory block was produced with as low as $1.7 \mathrm{~mL}$ which was insufficient to produce circumferential spread [16]. Therefore, extraneural, and even non-circumferential LA injection can produce a successful blockade.

Circumferential extraneural LA injection, however, appears to be more effective for regional block, particularly for onset of blockade. Brull et al. showed that popliteal sciatic block was more complete at 30 min with injection in three of four quadrants around the nerve compared to the same volume injected in one location. The blocks were not tested postoperatively, however, so it cannot be said that the single-quadrant blocks did not produce effective analgesia after more time had elapsed [17]. Later studies showed that popliteal sciatic block with LA injected deep to the paraneurium at the bifurcation, but outside the epineurium of the tibial and common peroneal nerves, resulted in faster onset to complete block than more proximal injection [18]. Popliteal injection deep to the paraneurium results in longitudinal spread of LA adjacent to the nerves for a number of centimeters. There is some disagreement about whether this injection location should be called extra- or intraneural, but since the LA spread is outside the outer epineurium of both the tibial and peroneal components of the sciatic nerve, extraneural seems the more appropriate terminology. Injection outside the paraneurium, by contrast, may spread radially into the popliteal fat rather than longitudinally along a length of nerve as needed for conduction block.

Each nerve block site may be different, though, because of tissue constraints to the flow of LA which are unique to each site. It may be likely that the ideal extraneural injection technique at the popliteal site is sub-paraneural, while at the femoral site, a subfascial injection deep to the fascia iliaca, but still outside the outer epineurium of the femoral nerve, may produce a circumferential and longitudinal spread around the nerve. For the brachial plexus, there is discussion about the identification of the actual outer epineurium versus the deep cervical fascia with respect to the naming of these connective tissue layers which surround the roots, trunks and divisions of the plexus. At the interscalene site, injection between the scalene muscles is sufficient to produce spread around the nerves, and an $\mathrm{ED}_{95}$ for surgical anesthesia using $0.75 \%$ ropivacaine injected through a catheter was identified as $7 \mathrm{~mL}$ [19]. Is this injection location extraneural and superficial to the outer epineurium of the brachial plexus, or intraneural? Orebaugh et al. performed ultrasound-guided interscalene needle placement between the hypoechoic brachial plexus roots or trunks in unembalmed cadavers, with injection of a small volume of India ink between elements of the plexus. On histologic exam, they found the injection had breached the outer epineurium in five of ten specimens, although there was no evidence of sub-perineural injection of ink [20].

This is a critical point-injection of LA deep to a layer of connective tissue surrounding the target nerve maintains a high concentration of LA in contact with a sufficient area of the nerve for conduction block; if this layer exists outside the outer epineurium (sciatic paraneurium or deep cervical fascia surrounding the brachial plexus), then breaching the outer epineurium is not necessary for a reliable block. This begs the question, though, as to whether these specific layers can be resolved or discriminated using current high frequency portable ultrasound machines commonly employed for regional block (see below).

\section{If Extraneural Injection is Usually Successful, Why Would a Clinician Choose to Perform Intraneural Injection?}

\section{Intraneural Injection is Not Always Harmful}

A number of studies and case reports since the advent of ultrasound-guided nerve block have shown that intraneural injection does not inevitably result in nerve injury. In the first such report, Bigeleisen studied axillary brachial plexus block and used a needle insertion endpoint of a "pop" or paresthesia while observing with ultrasound; he defined intraneural injection as one resulting in nerve swelling and/ or "halo" formation around the nerve with an initial $3 \mathrm{~mL}$ LA injection. In all 26 patients the blocks were successful, with a $69 \%$ incidence of intraneural injection, and no PONS at 1-week and 6-month follow-up [21]. In two investigations of nerve-stimulator-guided, ultrasoundmonitored popliteal block, an incidence of intraneural injection was reported in $66 \%$, and $94 \%$ of blocks, with no PONS $\left[6^{\bullet}, 22\right]$. Finally, in a larger study of 100 patients undergoing nerve stimulator-guided median nerve block, a pre- and post-block ultrasound evaluation showed a $43 \%$ incidence of intraneural injection; no patient had PONS up to one month postoperatively [23]. Although these studies provide some reassurance that intraneural injection (presumably extrafascicular) does not always cause injury, the 
number of patients is too small to conclude there is no risk to performing an intraneural injection.

The explanation for normal neurologic recovery following intraneural injection may be that most intraneural needle placements do not enter the fascicle. In the porcine study mentioned above, only $10 \%$ of intraneural needle placement under direct vision showed histologic evidence of perineural disruption [2]. In an in vitro sciatic nerve specimen from an unembalmed cadaver, $3 \%$ of fascicles had damage after transfixion using A-bevel needles [22]. Anatomic studies have shown a variable amount of connective tissue (inner epineurium) relative to fascicular tissue for different nerves, and also for different locations along a single nerve. Early neuroanatomic studies showed this with the sciatic and other peripheral nerves [24], and Moayeri et al. [25] have also demonstrated this finding using cryomicrotome sectioning along the brachial plexus. Certain locations for nerve block, then, may have a greater margin of safety for intentional intraneural needle placement to avoid fascicular injury at sites where the amount of inner epineurium is substantial.

Of some concern, however, there is preliminary evidence that even intraneural, but extrafascicular, injection may not be completely harmless. Such needle placement and LA injection in a porcine model with sacrifice and histologic exam at 7 days showed axonal degeneration in six of ten pigs, although there was no concurrent behavioral evidence of neural dysfunction [26]. Further, in an open sciatic nerve injection of ropivacaine $0.75 \%$ in rats, histologic damage was greatest with intraneural-intrafascicular injection, but was also greater with intraneuralextrafascicular injection than extraneural [27]. If these findings are confirmed by other investigators, they suggest the margin of safety for intraneural extrafascicular injection of LA may be less than extraneural placement.

\section{Intraneural Injection May Produce "Better" Nerve Block}

While it is clear that intraneural injection is probably not routinely damaging, there still must be some benefit for a clinician to select this needle insertion target. There is evidence that nerve blocks following intraneural injection may result in faster or even longer neural blockade. In the popliteal studies noted above, a more rapid onset to complete sensory blockade was shown with intraneural injection as evidenced by nerve swelling on ultrasound. It should be noted that a complete block also occurred in patients without swelling, but took more time [18]. Faster onset of block with intraneural injection was also shown for median nerve block, as evidenced by nerve swelling, but there was no statistically significant difference in the ultimate block success between patients with circumferential LA spread with, or without nerve swelling [23]. In a study of subgluteal sciatic block, where the anesthesiologist intended to inject extraneurally, but post-block video analysis showed $16.3 \%$ of needles were intraneural upon initial injection; those patients had faster onset of sensory and motor block. Again, there was no difference in ultimate block success (or duration) [28•].

In a similar post-procedure assessment of video-recorded ultrasound-guided interscalene or supraclavicular block using a high frequency transducer, Liu et al. [29] reported a nearly identical $17 \%$ incidence of unintended initial intraneural injection in 257 patients. Again, the block duration was the same between patients with intraneural or extraneural injection, and no patient demonstrated PONS.

Although the aforementioned studies have not shown longer duration with intraneural injection, there are case reports of unusually long blocks with intraneural injection [30]. In the above-noted study on extra- or intraneural injection in dogs, the intraneural-extrafascicular injections produced significantly longer blockade than the extraneural injections [10]. The longer "dwell time" of LA within the nerve, with higher local concentration of LA might explain prolonged block, but the clinical studies show this is not a predictable phenomenon.

Ultimately, even though intraneural injection may result in faster, and sometimes longer blocks, our current technology does not guarantee the clinician can safely position the needle intraneurally but reliably extrafascicularly. The lateral resolution of a linear, $10 \mathrm{MHz}$ ultrasound transducer is approximately $1 \mathrm{~mm}$, and many fascicles and interfascicular fibers are smaller than this diameter. For deeper blocks requiring low frequency transducers, the ability to discriminate small fascicles is even poorer. Although injection pressure monitoring, subjective, or objective, may provide additional feedback to indicate a possible intraneural intrafascicular needle position, injection must be aborted before flow occurs since fascicular damage may occur with exceedingly small volumes of LA. Conversely, a false positive rate of $8.3 \%$ was demonstrated with intraneural-extrafascicular injection in a porcine model using 25 psi as a pressure limit, and $20 \%$ using 15 psi. These pressures, if achieved during clinical block, should prompt needle repositioning before injection, but this study shows the needle could be extrafascicular [31].

\section{The Future}

The imaging resolution of ultrasound should continue to improve, and the ability to definitively resolve an intraneural, but predictably extrafascicular needle position, will be enhanced. Additionally, other technological advances with electrical impedance or pressure monitoring may help to assure an intraneural but extrafascicular needle position. 
It must be remembered, though, that animal investigation has suggested the margin of safety for even intraneuralextrafascicular LA injection may be lower than extraneural.

\section{Conclusions}

To summarize, with the use of ultrasound-guided regional block, the clinician may choose the site for deposition of LA to be either extraneural, particularly where fascial barriers will constrain the LA to spread around and along the nerve, or may choose to pierce the outer epineurium and deposit the LA intraneurally because of evidence of faster onset, or more prolonged blockade. Even with the extraneural site plan, there is a finite incidence of unintended needle advancement such that the epineurium is penetrated. Whether intentional or unintentional, the intraneural site brings the needle tip closer to breaching the perineurium, which carries a significant risk of PONS, and current ultrasound technology and resolution do not provide assurance that the clinician can distinguish intraneural-extrafascicular from intraneural-intrafascicular placement. The use of shortbevel needles less able to penetrate the perineurium, and the additional monitoring of patient response and injection pressure may decrease the risk of fascicular injury, as well as selection of a block site where the ratio of epineurium to neural tissue is high. However, there are no current methods which can reliably detect intrafascicular needle tip placement before damage may have occurred. Given this state of current technology, and since extraneural injection is consistently successful, the weight of evidence recommends against intentional intraneural needle placement at the present time. Successful block with the lowest achievable risk of PONS should always be the goal.

\section{Compliance with Ethics Guidelines}

Conflict of Interest Robert S. Weller declares that he has no conflict of interest.

Human and Animal Rights and Informed Consent This article does not contain any studies with human or animal subjects performed by any of the authors.

\section{References}

Papers of particular interest, published recently, have been highlighted as:

- Of importance

1. Winnie AP. Considerations concerning complications, side effects and untoward sequelae. In: Winnie AP, editor. Plexus anesthesia. Philadelphia: W.B. Saunders Co; 1993. p. 253-7.
2. Chan VW, Brull R, McCartney CJ, et al. An ultrasonographic and histological study of intraneural injection and electrical stimulation in pigs. Anesth Analg. 2007;104:1281-4.

3. Sinha SK, Abrams JH, Weller RS. Ultrasound-guided interscalene needle placement produces successful anesthesia regardless of motor stimulation above or below $0.5 \mathrm{~mA}$. Anesth Analg. 2007; 105:848-52.

4. Perlas A, Niazi A, McCartney C, et al. The sensitivity of motor response to nerve stimulation and paresthesia for nerve localization as evaluated by ultrasound. Reg Anesth Pain Med. 2006;31:445-50.

5. Bigeleisen PE, Moayeri N, Groen GJ. Extraneural versus intraneural stimulation thresholds during ultrasound-guided supraclavicular block. Anesthesiology. 2009;110:1235-43.

6. - Sala-Blanch X, López AM, Pomés J, et al. No clinical or electrophysiologic evidence of nerve injury after intraneural injection during sciatic popliteal block. Anesthesiology 2011;115:589-595. This study showed that during performance of nerve-stimulator-guided posterior popliteal sciatic block using the common endpoint of tibial response at 0.3-0.5 mA, post-block ultrasound showed evidence of intraneural injection in $94 \%$ of patients! Importantly, at 1 and 4 weeks post-block, no patient had clinical or electrophysiological evidence (nerve conduction studies) of nerve dysfunction.

7. Sunderland S. The connective tissues of peripheral nerves. Brain. 1965;88:841-54.

8. Selander D, Brattsand R, Lundborg G, Nordborg C, Olsson Y. Local anesthetics: importance of mode of application, concentration and adrenaline for the appearance of nerve lesions. An experimental study of axonal degeneration and barrier damage after intrafascicular injection or topical application of bupivacaine (Marcain). Acta Anaesthesiol Scand. 1979;23:127-36.

9. Rice AS, McMahon SB. Peripheral nerve injury caused by injection needles used in regional anaesthesia: influence of bevel configuration, studied in a rat model. Br J Anaesth. 1992;69: 433-8.

10. Kapur E, Vuckovic I, Dilberovic F, et al. Neurologic and histologic outcome after intraneural injections of lidocaine in canine sciatic nerves. Acta Anaesthesiol Scand. 2007;51:101-7.

11. Andersen HL, Andersen SL, Tranum-Jensen J. Injection inside the paraneural sheath of the sciatic nerve: direct comparison among ultrasound imaging, macroscopic anatomy, and histologic analysis. Reg Anesth Pain Med. 2012;37:410-4.

12. Nagda SH, Rogers KJ, Sestokas AK, et al. Neer Award 2005: peripheral nerve function during shoulder arthroplasty using intraoperative nerve monitoring. J Shoulder Elbow Surg. 2007; $16:$ S2-8.

13. Neal JM, Bernards CM, Hadzic A, et al. ASRA practice advisory on neurologic complications in regional anesthesia and pain medicine. Reg Anesth Pain Med. 2008;33:404-15.

14. Raymond SA, Steffensen SC, Gugino LD, Strichartz GR. The role of length of nerve exposed to local anesthetics in impulse blocking action. Anesth Analg. 1989;68:563-70.

15. O'Donnell BD, Iohom G. An estimation of the minimum effective anesthetic volume of $2 \%$ lidocaine in ultrasound-guided axillary brachial plexus block. Anesthesiology. 2009;111:25-9.

16. Latzke D, Marhofer $P$, Zeitlinger $M$, et al. Minimal local anaesthetic volumes for sciatic nerve block: evaluation of ED 99 in volunteers. Br J Anaesth. 2010;104:239-44.

17. Brull R, Macfarlane AJ, Parrington SJ, Koshkin A, Chan VW. Is circumferential injection advantageous for ultrasound-guided popliteal sciatic nerve block?: a proof-of-concept study. Reg Anesth Pain Med. 2011;36:266-70.

18. Sala-Blanch X, de Riva N, Carrera A, et al. Ultrasound-guided popliteal sciatic block with a single injection at the sciatic division results in faster block onset than the classical nerve stimulator technique. Anesth Analg. 2012;114:1121-7. 
19. Vandepitte C, Gautier P, Xu D, Salviz EA, Hadzic A. Effective volume of ropivacaine $0.75 \%$ through a catheter required for interscalene brachial plexus blockade. Anesthesiology. 2013;118: 863-7.

20. Orebaugh SL, McFadden K, Skorupan H, Bigeleisen PE. Subepineurial injection in ultrasound-guided interscalene needle tip placement. Reg Anesth Pain Med. 2010;35:450-4.

21. Bigeleisen PE. Nerve puncture and apparent intraneural injection during ultrasound-guided axillary block does not invariably result in neurologic injury. Anesthesiology. 2006;105:779-83.

22. Sala-Blanch X, Ribalta T, Rivas E, et al. Structural injury to the human sciatic nerve after intraneural needle insertion. Reg Anesth Pain Med. 2009;34:201-5.

23. Dufour E, Cymerman A, Nourry G, et al. An ultrasonographic assessment of nerve stimulation-guided median nerve block at the elbow: a local anesthetic spread, nerve size, and clinical efficacy study. Anesth Analg. 2010;111:561-7.

24. Sunderland S. Anatomical features of nerve trunks in relation to nerve injury and nerve repair. Clin Neurosurg. 1970;17:38-62.

25. Moayeri N, Bigeleisen PE, Groen GJ. Quantitative architecture of the brachial plexus and surrounding compartments, and their possible significance for plexus blocks. Anesthesiology. 2008;108: 299-304.

26. Lupu CM, Kiehl TR, Chan VW, et al. Nerve expansion seen on ultrasound predicts histologic but not functional nerve injury after intraneural injection in pigs. Reg Anesth Pain Med. 2010;35: $132-9$.

27. Whitlock EL, Brenner MJ, Fox IK, et al. Ropivacaine-induced peripheral nerve injection injury in the rodent model. Anesth Analg. 2010;111:214-20.

28. • Hara K, Sakura S, Yokokawa N, Tadenuma S. Incidence and effects of unintentional intraneural injection during ultrasoundguided subgluteal sciatic nerve block. Reg Anesth Pain Med 2012;37:289-293. This study is important in demonstrating that clinicians intending to perform an extraneural needle placement will sometimes instead penetrate the outer epineurium, and this intraneural injection appears to result in faster onset of blockade. The success and duration of block were not different, however, and no patient had PONS.

29. Liu SS, YaDeau JT, Shaw PM, et al. Incidence of unintentional intraneural injection and postoperative neurological complications with ultrasound-guided interscalene and supraclavicular nerve blocks. Anaesthesia. 2011;66:168-74.

30. Benhamou D, Blonski E, Lévy P, Plessis E, Chalhoub V. Ultralong duration of a peripheral nerve block: a possible consequence of intraneural (subepineural) local anaesthetic injection. Ann Fr Anesth Reanim. 2010;29:589-91.

31. Altermatt FR, Cummings TJ, Auten KM, et al. Ultrasonographic appearance of intraneural injections in the porcine model. Reg Anesth Pain Med. 2010;35:203-6. 